\title{
Policy Analysis of Traditional Chinese Medicine Health Service in China Based on Policy Tools
}

\author{
${ }^{1}$ Jiangxi University of Traditional Chinese Medicine, Nanchang, Jiangxi, China \\ ${ }^{2}$ Jiangxi University of Traditional Chinese Medicine, Nanchang, Jiangxi, China \\ ${ }^{3}$ Jiangxi University of Traditional Chinese Medicine, Nanchang, Jiangxi, China \\ ${ }^{4}$ Jiangxi University of Traditional Chinese Medicine, Nanchang, Jiangxi, China \\ ${ }^{5}$ Jiangxi University of Traditional Chinese Medicine, Nanchang, Jiangxi, China \\ ${ }^{*}$ Corresponding author. Email: 2439677356@qq.com.
}

Jin Jiaqi ${ }^{1}$, Wang Junyong ${ }^{2 *}$, Jian $\mathrm{Wu}^{3}$, Luo Renwen $\mathrm{Ya}^{4}$, Ding $\mathrm{Lan}^{5}$

\begin{abstract}
Purpose: In order to combine the current selection and application of medical health service policy tools in China, through the results of data analysis, it provides relevant basis for the formulation and implementation of Chinese medicine health service policies in China. Methods: Based on Rothwell and Zegveld policy tools. The policy documents included in the analysis were coded and extracted, and the policy tools of 37 collected Chinese medicine policy documents were classified and statistically analyzed by X-dimension analysis. Results: In the collected Chinese medicine health service policies, environmental policy tools account for about $46 \%$, and supply policy tools about $44 \%$ demand-based policy tools about $10 \%$. Conclusion: It is necessary to select and standardize the policy tools of TCM health service systematically and scientifically, further optimize the structure ratio of basic policy tools, promote the rational use of basic policy tools in various sub-sectors of health service industry, and further improve the targeted accuracy of the use of policy tools [1].
\end{abstract}

Keywords: Chinese medicine health service, Policy tools, Policy analysis

\section{BACKGROUND}

Chinese medicine is the most unique health security service resource in China at present. Accelerating the construction of Chinese medicine health service industry is one of the main tasks to promote the development of health security service industry and safeguard the health of the broad masses of the people. In recent years, the state has issued a series of policies on revitalizing and developing the cause of traditional Chinese medicine, which has become a powerful guide and support for the healthy development of the cause of traditional Chinese medicine in China. These policies will also promote the health management and service of traditional Chinese medicine in China more comprehensively, deeply and scientifically. Policy tools are the intermediary elements that determine policy objectives and implementation results.

Policy tools, also known as government tools or governance tools, refer to social authorities to solve social public problems or achieve specific policy goals or results, Select and determine a series of means, behavioral methods or mechanisms that act on policy objectives[2]. Policy instruments serve as a bridge between policy objectives and implementation outcomes, At present, China's traditional Chinese medicine health management service lacks policy tools to analyze it in the field of policy research. Therefore, this paper will discuss this field of traditional Chinese medicine health management service through policy tools.

\section{INFORMATION AND METHODOLOGY}

\subsection{Sources}

The title or key words of this study are "Chinese Medicine", "Chinese Medicine Health", "Chinese Medicine Health Service", "Chinese Medicine Development", "13th Five-Year Plan of Chinese Medicine", "Chinese Medicine Innovation" and "Key Points of Chinese Medicine Work", search the laws, regulations, plans, opinions, methods, notices, plans and other documents reflecting government policies published by the websites of relevant ministries and agencies of the State Council from 2007 to 2019. 


\subsection{Criteria For Inclusion And Exclusion}

Inclusion criteria: (1) The issuing institution is the State Council, Ministry of Health/National Health and Family Planning Commission/National Health and Health Commission, National Administration of Traditional Chinese Medicine and other relevant ministries and agencies of the State Council. (2) the policy content includes information related to traditional Chinese medicine health services. (3) The types of policies are laws and regulations, plans, opinions, methods, notices, Programs, etc. (4) The policy was issued from January 1, 2007 to December 31, 2019.

Exclusion criteria: There are only key words in the document but no substantive policy content.

\subsection{Research Methodology}

\subsubsection{Classification And Definition Of Policy Tools}

Classification and Definition of Chinese Medicine Health Services are influenced by three important factors: medical service providers (suppliers), users (demanders), medical systems and policy guidance (environment). Therefore, this article takes Rothwell and Zegveld's policy tool model as the viewpoint[3]The classification criteria are supply-oriented policy tools, demand-oriented policy tools and environmental-oriented policy tools.

(1) Supply-oriented policy tools are mainly embodied in the policy tools that the government effectively promotes the development of traditional Chinese medicine health services from the perspective of suppliers by directly providing support and guarantee for the traditional Chinese medicine health service industry. Specifically, it includes institution building (focusing on the establishment of institutions), infrastructure, financial support, supporting services, etc.

(2) Demand-based policy tools are mainly reflected in the government taking various measures to reduce market barriers and risks in the development of traditional Chinese medicine health care services. Release the needs of residents and drive the industry to be fast and steady Land development, mainly including government Subsidies, service outsourcing, demonstration/ pilot projects, overseas promotion and other measures.

(3) The main function of environmental policy tools is to act on the whole health service system of traditional Chinese medicine. Its characteristic is that the government can innovate and improve the external environment and conditions of the industry. It lays a solid foundation for the construction and prosperity of Chinese medicine health services and directly promotes the industry progress of Chinese medicine health services, which mainly including the government through laws and regulation, deregulation, policy advocacy, tax concessions, medical insurance support, etc.

\subsubsection{The Content Analysis Of Policy Tools}

Sorts the included policy texts in chronological order, and codes the policy units of the policy texts according to "policy number-corresponding policy tools", classification: When the policy text appears repeatedly in the same chapter, the number will be calculated separately when it is included in the analysis. Finally, all codes are included in Excel 2019 for frequency statistics.

\section{RESULTS}

\subsection{Basic Information On The Policy Text}

37 Chinese medicine policy documents were included in the analysis according to the number of items, and environmental policy tools accounted for approx. (46\%) Supply-oriented policies account for about $44 \%$, demandbased policy tools account for about $10 \%$. In terms of issuing agencies, 29 were issued by National Administration of Traditional Chinese Medicine alone or jointly with other departments, and 7 were jointly issued by government agencies of many countries. In terms of the year of publication, the largest number of policy documents on Chinese medicine health services were issued in 2016 (10times), followed by 2017 (5times).

\subsection{Use Of Policy Tools}

In environmental policy, regulation, policy propaganda, as well as organizational coordination, the ratio of legal control is as high as $67 \%$, while the other two ratios are $7 \%$ and $7 \%$ respectively. Among environmental policies, the proportion of financial support and medical insurance support is relatively low (both 1\%). This shows to some extent that, At present, Chinese medicine still tends to publicize laws and regulations, to show their "unique" influence, however, according to the data collected by environmental policy tools, Traditional Chinese medicine health service still lacks a certain understanding of its real improvement of service quality and competitiveness, It is impossible to really improve the overall level of Chinese medicine health services. At the same time, the frequency of use of policy tools such as medical insurance and financial support is relatively low, which is not conducive to the good development of Chinese medicine health services.

In the supply-oriented policy, the means of scientific and technological support is used the highest number of times, 220 times, accounting for about $35 \%$. The number of times talent support is used as a policy tool is 97 , accounting for about $16 \%$. Information support (promoting the development of the industry by improving the level of informatization, such as strengthening the informatization construction of hospitals and traditional 
Chinese medicine health service institutions, improving information systems, etc.) was mentioned 80 times, accounting for about $13 \%$.The tool of institution building (improving the health service network and system of traditional Chinese medicine and speeding up the construction of relevant departments and teaching and research institutions) has also been mentioned 68 times, accounting for $11 \%$ of the total number of supplyoriented policies, while the proportion of infrastructure is slightly lower (about $4 \%$ ), and the proportion of financial support is the lowest among all supply-oriented policy tools, only $0.3 \%$. These data show that during this period of time, the government supported and optimized a series of technologies related to Chinese medicine, and improved the informationization level of Chinese medicine industry. However, at the same time, some long-standing problems have been found in the data, such as: the government has less investment in basic construction such as housing, basic equipment and facilities to ensure the basic conditions needed for the development of the industry, and the government has less financial support for traditional Chinese medicine health services.

Among the demand-based policies, pilot/demonstration projects and overseas promotion have the highest mention rates, reaching 80 times and 50 times respectively, accounting for $56 \%$ and $35 \%$ respectively. Service outsourcing (the government vigorously promotes Chinese medicine health services and their integration with other industries, and develops related supporting industries) was mentioned five times, accounting for about $3 \%$. Financial subsidies, government procurement and other policy tools are used relatively rarely, accounting for $2 \%$ and $1 \%$ of the overall demand-based policies respectively. According to the data reflected by policy tools, the government is expanding pilot/demonstration projects with Chinese medicine characteristics, expanding foreign trade and investment, promoting Chinese medicine technology, industry going out has expanded the influence of Chinese medicine health services. However, how to promote the development of Chinese medicine health services through financial subsidies and government procurement still has more resistance in the current policy formulation and cannot become the main force of the current Chinese medicine development policy.

\section{CONCLUSION}

\subsection{More Attention Should Be Paid To Reasonable And Even Distribution In The Use Of Policy Tools}

From the perspective of scale analysis and rational use of policy tools, the use frequency of environmental policy tools is much higher than that of demand-based policy tools, although it reflects the role of the Chinese government in promoting the development and construction of Chinese medicine health services. However, while actively developing the platform, we should not neglect the use of other policy tools. When using TCM policy tools in the future, the government should appropriately reduce the use of environmental policy tools and focus on improving the use of demandbased and supply-based policy tools.

\subsubsection{Optimize The Supply-Oriented Policy And Increase The Flow Of Traditional Chinese Medicine Infrastructure}

In the process of optimizing the supply-oriented policy, Increase the advanced talents of Chinese medicine, Chinese medicine financial support systemAttach importance to. In terms of strengthening the support of Chinese medicine talents, the state and government departments need to intensify the research on Chinese medicine professional technology and personnel, Analyze the characteristics and advantages of Chinese medicine, clarify the "unique positioning" of Chinese medicine in health management and service industry, and constantly inherit and innovate the "core technology" of Chinese medicine health management, Establish a talent training system with the uniqueness of Chinese medicine, and train Chinese medicine talents to meet the current social needs. In strengthening financial support, The government should increase capital investment, establish a reasonable financial capital growth mechanism, Implement special funds for the development of Chinese medicine, Improve the financial guarantee for the development of Chinese medicine, especially increase the financial assistance for the development of Chinese medicine in rural areas and poverty-stricken areas. At the same time, it is suggested that the government should vigorously develop the Chinese medicine health service network and improve the Chinese medicine information system, Reasonable allocation of traditional Chinese medicine service facilities, equipment and personnel, give full play to the advantages and functions of traditional Chinese medicine in basic health work [4].

\subsubsection{Increase The Proportion Of Demand- Based Policy Tools Used To Stimulate The Demand For Traditional Chinese Medicine Health Services}

The government should strengthen the potential tapping work on the demand side. Taking demand as the starting point, we should actively connect with demand, promote the structural reform on the supply side, make the contents, methods, tools and technologies of Chinese medicine health services more "affinity" of residents, and have the ability to deal with and solve practical problems. At the same time, we should intensify the crackdown on "pseudo-Chinese medicine", thus forming a good demand environment. 


\subsection{In Terms Of Regulatory Policies, The Market And The government Need To Strengthen Their Responsibilities And Clarify The Division Of Functions}

From the perspective of the use of policy tools for Chinese medicine health service policies, on the one hand, attention should be paid to the use of two core tools: legal control and policy propaganda. Attach importance to the construction of core resources such as scientific and technological support and personnel training and education. However, what should and should not be managed by the market and the government has not been really clarified, and the work list has not been divided by category according to clear, clear and operable responsibilities. The problems of vacancy, dislocation and offside are still very obvious.

\subsubsection{Adjust The Internal Structure And Clarify The Division Of Functions Between The Market And The Government.}

For environmental policy tools, attention should be paid to the adjustment of their internal structure. It is necessary to clarify the division of responsibilities between the government and the market in the development of Chinese medicine health services, gradually establish a dynamic adjustment mechanism of government functions, and form a clear, clear, measurable and dynamic "list of government functions", so that invisible hands and market allocation can play a major role and match more reasonably.

\subsection{The Efficiency Of Policy Uploading And Distribution And The Implementation Of Various Administrative Regions Need To Be Improved}

In the propaganda of Chinese medicine health service policy, policy propaganda is a good thing, but if the policy is not implemented due to knowledge propaganda and just shouting slogans and doing nothing, it will also seriously affect the image of Chinese medicine in the eyes of the people of the whole country and seriously hinder the sustained and healthy development of Chinese medicine. The government's thinking and thinking on the training of talents related to the cause of traditional Chinese medicine are not clear, the government's execution is seriously insufficient, and there are obvious defects in the identification, homogeneity and quality of the trained talents, resulting in some talents trained at this stage unable to meet the needs of the society and unable to provide qualified and excellent traditional Chinese medicine health services for the society. On the other hand, at this stage, the Chinese government still does not pay enough attention to the important work of the development of traditional Chinese medicine infrastructure construction, financial support and financial support, medical insurance support and financial subsidies, government procurement services and other industries.

\subsubsection{The government and departments should carry out specific responsibilities and strengthen the implementation.}

The government and relevant departments need to shoulder their responsibilities, attach importance to the publicity of policies, and carry out policy publicity activities in various ways. Strengthen the construction of quality evaluation system for the development of traditional Chinese medicine, analyze and solve the current situation and problems of the development of traditional Chinese medicine. It is suggested that the government should increase financial support for the development of Chinese medicine, encourage commercial banks to open up green credit channels and lower loan interest rates, increase credit supply[5]In terms of medical insurance support, It is suggested that qualified Chinese medicine service items should be included in the payment scope of basic medical insurance, and the reimbursement ratio and reimbursement scope of Chinese medicine service items with higher results should be appropriately increased to simplify reimbursement procedures[6].

\section{REFERENCES}

[1] Luo Xian, Ranna Ahati, Li Ruifeng.Quantitative analysis of Beijing's health service policy based on the perspective of policy tools [J]. China Health Policy Research, 2020, 13 (07): 66-71.

[2] Wang Yibao, Cao Ming. Public Policy Analysis [M]. Jiangsu: China's mining industry

[3] MICHAEL H, M RAMESH, ANTHONY P. Student pub-lic policy: Policy cycles and policy subsystems [M]. Bos-ton: Oxford University Press, 2003: 87

[4] Gao Jianan, Hong Xuezhi, Lareina C, Xu Fuqin, Pu Jin, Li Xue, Pei Ye, Kong Junhui.Feasibility analysis of incorporating traditional Chinese medicine into basic public health services [J]. Medicine and Society, 2016, 29 (11): 28-30.

[5] Lu Xueyan, Chen Jie, Chen Xiuhong, Zuo Bo.Practice and Thinking of Financial Institutions Supporting the Optimization and Upgrading of Local IndustriesTaking Tonghua City, Jilin Province as an Example to Support Traditional Chinese Medicine Industry [J]. Credit Investigation, 2016, 34 (07): 87-89.

[6] Song Xiaoqing, Xiong Jixia, Yao Yunan.Research on Chinese medicine policy based on policy tools [J]. Medicine and Society, 2019, 32 (11): 4-8. 